Artykuły

Studia luridica Lublinensia vol. XXVI, 1, 2017

DOI: 10.17951/sil.2017.26.1.457

\author{
Ángel Martínez Gutiérrez \\ Universidad de Jaén \\ martinez@ujaen.es
}

\title{
La tutela de las Denominaciones Geográficas en la Unión Europea. Luces y Sombras
}

Ochrona oznaczeń geograficznych w Unii Europejskiej. Blaski i cienie

\section{RESUMEN}

Las indicaciones geográficas ofrecen a los sujetos caules comercializan al mercado europeo sus productos de agricultura una forma juridica muy compleja y segura. Hay que tener en cuenta que este tipo de proteccion de los productos agroalimentarios, por un lado genera muchas ventajas como por ejmplo la posibilidad de promocionar su producto en el mercado de la Union Europea y distinguirlo entre los todos, pero por otro lado a veces complica la situacion del empresario. El objeto de este articulo es dereminar cuales son las ventajas y desventajas de proteger sus productos agroalimentarios via las indicaciones geograficas.

Palabras clave: indicaciones geográficas; promoción; tutela

\section{LA IMPORTANCIA DE LAS INDICACIONES GEOGRÁFICAS EN LA PROMOCIÓN COMERCIAL DE LOS PRODUCTOS O SERVICIOS}

\section{Introducción}

Como es sabido, las indicaciones geográficas son aquellos signos o símbolos que permiten la designación explícita o implícita de un determinado lugar. Se trata de una noción sumamente amplia que aglutina no sólo a las denominaciones geográficas en sentido estricto (esto es, los topónimos), sino también a aquellas otras indicaciones que, sin aludir de forma directa y elocuente a una zona determinada, permiten realizar un proceso de asociación en la mente del público de los consumidores que facilita, desde luego, tal deducción. Éste sería el caso, por 
ejemplo, de los colores de una bandera, un monumento típico o, incluso, una denominación en un idioma concreto.

Pues bien, ha de destacarse en esta sede la relevancia de estas indicaciones geográficas en el ámbito comercial de los productos o servicios, al manifestarse en la práctica como un instrumento de promoción especialmente útil. Y es que, como consecuencia de su capacidad para comunicar un cúmulo de informaciones en el tráfico económico, la inclusión de una indicación geográfica en la presentación de una oferta empresarial concreta permite no sólo la reducción del esfuerzo del público de los consumidores en el proceso de aprendizaje y posterior recuerdo de la misma, sino también la disminución de los costes económicos y temporales en publicidad que el empresario debe soportar para conseguir que los elementos formales insertos en la presentación de la oferta empresarial concreta transmitan un mensaje sobre las características de los productos o servicios².

Obsérvese, en este sentido, cómo la vinculación de la presentación de un producto o servicio a un ámbito geográfico determinado, atenuando los efectos negativos de la asimetría informativa existente en el mercado, permite el suministro de importante y variada información al público de los consumidores que, desde luego, puede desencadenar el acto de compra. En efecto, la inclusión de una indicación geográfica en la promoción de una oferta empresarial concreta puede informar, por ejemplo, sobre el origen efectivo de los productos y, en su caso, sobre sus características cualitativas, pero también, sin llegar a este extremo, podría provocar unos sentimientos positivos que, sugestionando a los destinatarios de la oferta empresarial concreta, condicionan su comportamiento económico y determinan finalmente la adquisición de la misma ${ }^{3}$.

1 Vide sobre las indicaciones geográficas indirectas, C. Fernández-Nóvoa, La protección internacional de las denominaciones geográficas de los productos, Madrid 1970, p. 2. Más recientemente se han ocupado de ellas, M. Botana Agra, Las denominaciones de origen, Madrid 2001, p. 19; M.M. Gómez Lozano, Denominaciones de origen y otras indicaciones geográficas, Cizur Menor 2004, pp. 25 y sigs.; G. Sena, Il diritto dei marchi. Marchio nazionale e marchio comunitario, $5^{\text {a }}$ edic., Milán 2007, p. 93, quien se refiere a la información geográfica derivada de la utilización de una palabra en idioma extranjero.

2 Vide: C. Fernández-Nóvoa, Fundamentos de derecho de marcas, Madrid 1984, pp. 23 y sigs., con relación a las marcas expresivas; idem, Tratado de Derecho de Marcas, $2^{\mathrm{a}}$ edic., Madrid 2004, pp. 191 y sigs.; J.A. Gómez Segade, La protección constitucional de la marca y de las denominaciones de origen, "ADI" 1981, Vol. 7, p. 312. En el mismo sentido parece deponer, Y. Plasseraud, Choisir, protéger et gerer vos marques, París 1977, pp. 51 y sigs.

3 Vide: M. Ricolfi, I fatti costitutivi del diritto al marchio. I soggetti, [en:] P. Auteri, G. Floridia, V. Mangini, G. Olivieri, M. Ricolfi, P. Spada, Diritto Industriale, Proprietà intellettuale e concorrenza, $2^{\text {a }}$ edic., Turín 2005, pp. 77 y 78; S. Magelli, Marchio e nome geografico, [en:] Studi di Diritto Industriale in onore di Adriano Vanzetti. Proprietà intellettuale e concorrenza, Vol. 2, Milán 2004, p. 910; N. Abriani, G. Cottino, M. Ricolfi, Diritto Industriale, Trattato di Diritto Comerciale, Vol. 2, Turín 2001, p. 19, quien sostiene la importancia promocional y publicitaria de las indicaciones geográficas. 


\section{Información suministrada por las indicaciones geográficas}

La importancia promocional de las indicaciones geográficas deriva principalmente de la información susceptible de ser comunicada a través de ella. Y en este sentido, si atendemos a los distintos nexos que pueden entablarse entre los productos ofertados bajo una indicación geográfica y el territorio referido por ésta, pueden diferenciarse, con carácter general, varios supuestos, cuya exposición realizamos de mayor a menor vinculación entre ambas variables. En efecto, por una parte, es posible que el lugar designado refiera a la zona de extracción, cultivo, elaboración o fabricación del producto que, por efecto de los componentes climáticos y humanos existentes en ella, podrían incluso determinar unos caracteres específicos y peculiares del producto respecto de otros del mismo género. Radica aquí, por tanto, el criterio que tradicionalmente ha permitido clasificar las denominaciones geográficas descriptivas de los productos en indicaciones de procedencia y denominaciones de origen, pero que en la actualidad, como consecuencia de la regulación comunitaria de las denominaciones geográficas protegidas, ha quedado finalmente superado. En efecto, en atención al contenido del régimen jurídico comunitario de las denominaciones geográficas protegidas, creemos que el grado de intensidad existente entre el origen geográfico del producto y sus características particulares podría determinar la diferenciación de una triple tipología de denominaciones geográficas descriptivas; a saber, denominaciones de origen, indicaciones geográficas protegidas e indicaciones de procedencia ${ }^{4}$.

Por otra parte, es posible que el lugar citado en la indicación geográfica, no presentando ninguno de los vínculos anteriormente citados y revelándose en la práctica como una indicación de fantasía, pueda facilitar no obstante la sugestión del público de los consumidores en orden a las características presentadas por los productos o servicios ofertados. Piénsese, por ejemplo, en la evocación provocada por las denominaciones Siberia y Tropical para diferenciar o publicitar equipos de congelación y sistemas de calefacción, respectivamente ${ }^{5}$.

${ }^{4}$ La doctrina científica italiana propone una nueva sistematización a la vista de la clasificación contenida en el Reglamento comunitario actualmente derogado. Véase, por ejemplo, M. Libertini, Indicazioni geografiche e segni distintivi, "Riv. Dir. Comm." 1996, Vol. 1, pp. 1057 y sigs., quien distingue entre indicaciones geográficas simples y cualificadas en razón de que comuniquen sólo una información sobre el origen o añadan además un mensaje sobre las características de los productos. En el mismo sentido, pueden consultarse, por ejemplo, J. Mota Maia, Marcas, indicaciones geográficas y denominaciones de origen en el derecho comunitario, [en:] A. Bercovitz Rodriguez-Cano, Marca y Diseño comunitarios, Cizur Menor 1996, pp. 43 y sigs.; M. Botana Agra, op. cit., pp. 24 y 25.

5 Así parece mostrarse, A. Casado Cerviño, La nueva Ley española de marcas, nombre comercialy rótulo de establecimiento. Razones para una reforma. Concepto de marca. Signos, "AC" 1990, pp. 605, quien sostiene que "el empresario suele pretender vincular ciertas características geográficas (calidad, clima, etc.) evocadas por el signo con los productos (o servicios) que va a distinguir $[\ldots]$.. 
Pero además, cuando la separación entre el lugar indicado y los productos resulta tal que no es posible entablar ninguna de las conexiones citadas anteriormente, puede ocurrir, no obstante, que la belleza, la historia, las tradiciones del territorio provoquen unos sentimientos positivos en el público de los consumidores que ocasionan la adquisición de la oferta empresarial diferenciada. Debe subrayarse por ello la función promocional presentada en este punto por las indicaciones geográficas, toda vez que, aun cuando no describan al producto ofertado bajo las mismas, facilitan su comercialización en todo caso ${ }^{6}$.

\section{LA APROPIACIÓN EN EXCLUSIVA DE LAS INDICACIONES GEOGRÁFICAS. VIABILIDAD Y SUPUESTOS}

\section{Introducción}

Dado que la referencia geográfica en la presentación de una oferta empresarial constituye uno de los instrumentos promocionales más simples y, a su vez, más efectivos de los empleados en el mercado para hacerse con el favor del público de los consumidores, resulta constatable en la práctica comercial una tendencia a la apropiación en exclusiva de las indicaciones geográficas a través de los diferentes títulos de Derecho industrial. Para comprobar esta circunstancia, basta observar el mercado de cualquier producto (principal aunque no exclusivamente de carácter agroalimentario), para corroborar que las indicaciones geográficas utilizadas en la presentación publicitaria del mismo han sido tutelada, por ejemplo, a través de la

${ }^{6}$ C. Fernández-Nóvoa, La adopción de un nombre geográfico extranjero como marca "ADI" 1975, Vol. 2, p. 351, quien afirma que la adopción de una denominación geográfica extranjera puede realizarse con la finalidad "de asociar las mercancías de la empresa a países y lugares que aun no poseyendo un especial renombre respecto de tales mercancías, son, sin embargo, altamente estimados por el público como consecuencia de la difusión de determinadas modas, corrientes de opinión, sucesos de actualidad, etc.”. En el mismo sentido se muestran, J. Massaguer Fuentes, Marca (Derecho Mercantil), "EJB”, Vol. 3, Madrid 1995, p. 4177; S. Magelli, op. cit., p. 911, quien sostiene que con el uso del nombre geográfico se desea "indicare una provenienza, o promettere una qualità, esercitare o evocare un fascino oppure tutte queste cose insieme [...]"; G. Ivone, Diritto alla denominazione e marchio geográfico, "Riv. Dir. Comm." 1993, Vol. 2, p. 464. Por su parte, el Tribunal de Justicia de las Comunidades Europeas parece sostener este mismo planteamiento en la Sentencia de 4 de mayo de 1999 (C-108/97 y C-109/97), dictada en el asunto Windsurfing Chiemsee, disponible en la website www.curia.eu.int, cuando justifica el interés en preservar la disponibilidad de la indicación geográfica controvertida en "su capacidad no sólo para revelar, llegado el caso, la calidad, y demás propiedades de las categorías de productos consideradas, sino también para influir de diversos modos en las preferencias de los consumidores, por ejemplo, vinculando los productos a un lugar que puede inspirar sentimientos positivos [...]" (fundamento jurídico núm. 26). Esta sentencia ha sido objeto de estudio específico en nuestro trabajo, A. Martínez Gutiérrez, La conformación de la marca de empresa con denominaciones geográficas, “ADI" 1999, Vol. 20, pp. 665 y sigs. 
inscripción de una marca de empresa o, en el mejor de lo casos, mediante el reconocimiento administrativo de una denominación geográfica protegidas. Incluso, cabe citar en esta sede los supuestos en que la indicación geográfica se integra en la estructura de la denominación social de la sociedad mercantil, cuyo objeto social refiere la producción, distribución o comercialización de un producto concreto.

Sin embargo, mientras que la inclusión de una indicación geográfica en un título de Derecho industrial de carácter colectivo no parece plantear en principio inconveniente alguno, su apropiación en exclusiva mediante un título de carácter individual suscita complejas interesantes cuestiones que vienen siendo resaltadas tradicionalmente por la doctrina científica y jurisprudencial española y extranjera. Y es que, además del eventual carácter descriptivo que puede presentar el signo concreto, el uso exclusivo y excluyente que deriva de la inscripción registral del mismo viene a casar mal con la naturaleza colectiva que, como es sabido, define a estas indicaciones geográficas, de tal manera que, junto al monopolio injustificado a favor del titular del signo individual ${ }^{7}$, se puede generar un riesgo de engaño en el público de los consumidores sobre el verdadero significado de la indicación geográfica ${ }^{8}$.

\section{Supuestos de apropiación en exclusiva}

Pues bien, la posibilidad de una apropiación en exclusiva de estos signos colectivos de carácter geográfica se ancla en la existencia de instrumentos concurrenciales que pueden ser conformados lícitamente con nombres geográficos. Éste es

${ }^{7}$ Se trata de un efecto negativo que ha sido expuesto de forma explícita por el artículo $11.4^{\circ}$ del Codice della proprietà industriale italiano (aprobado por Decreto legislativo núm. 30, de 10 de febrero de 2005) cuando, regulando la viabilidad de las marcas colectivas geográficas, ordena que "(i)n tale caso, peraltro, l'Ufficio italiano brevetti e marchi può rifiutare, con provvedimento motivato, la registrazione quando i marchi richiesti possano creare situación di ingiustificato privilegio o comunque recare pregiudizio allo sviluppo di altre analoghe iniziative nella regione [...]". Sobre el alcance de este precepto, pueden consultarse, por ejemplo, G. Floridia, Il riassetto della proprietà industriale, Milán 2006, pp. 80 y sigs.; G. Ghidini, F. De Benedetti, Codice della proprietà industriale. Commento alla normativa sui diritti derivanti da brevettazione e registrazione, $1^{\text {a }}$ edic., Milán 2006, pp. 28 y sigs. Radica aquí la razón que justifica la tradicional admisión de la conformación de marcas geográficas en los casos donde no es posible que se verifique una situación del género. $\mathrm{Y}$, en este sentido, es paradigmático el supuesto conocido con el nombre "propiedad del empresario" que alude al supuesto en que el territorio designado con la indicación geográfica pertenece en exclusiva al titular marcario. Vide: C. Fernández-Nóvoa, Fundamentos de derecho de marcas, pp. 141 y sigs.; J. Massaguer Fuentes, op. cit., p. 4176; S. Magelli, op. cit., pp. 921 y 922; M. Libertini, op. cit., pp. 1051 y 1054; A. Nervi, Le denominazioni di origine protette e marchi: spunti per una ricostruzione sistematica, "Riv. Dir. Comm." 1996, Vol. 1, pp. 889 y 890.

${ }^{8}$ Vide: C. Fernández-Nóvoa, La adopción de un nombre..., p. 348; idem, Fundamentos de derecho de marcas, pp. 163 y 164; G. Ghidini, F. De Benedetti, op. cit., p. 32; C. Heaht, Il caso Budweiser, "Riv. Dir. Ind." 2004, Vol. 2-3, pp. 89 y 90, quien añade los problemas de falta de capacidad distintiva si la inscripción como marca la realiza una persona ubicada en la zona referida; S. Magelli, op. cit., pp. 912 y 913; M. Libertini, op. cit., pp. 1034 y 1035. 
el caso, por ejemplo, del signo distintivo por excelencia - esto es, la marca - que, estando llamada a diferenciar los productos o servicios de una empresa de aquellos otros con procedencia empresarial diferente no marcados o marcados con signo diverso, pueden estar construidos con indicaciones geográficas directa o indirecta. Así lo recoge, por ejemplo, el artículo $7.1^{\circ} \mathrm{c}$ ) del Reglamento de Marca Comunitaria.

Igualmente, ocurre con las denominaciones sociales. Y es que, aun cuando vienen a diferenciar en el tráfico económico a sujetos de derecho de carácter social, lo cierto es que, en la práctica comercial, ha manifestado un importante valor comunicativo, lo que ha provocado que también sean aprovechadas para vincular una oferta empresarial a una información geográfica determinada.

Otro tanto puede decirse de los nombres de dominio, puesto que, si bien permiten la identificación de un sujeto en sede electrónica, suele incluirse también información geográfica en su estructura.

Es evidente, por tanto, que el Ordenamiento jurídico ofrece a los empresarios diferentes vías de diferenciación en el mercado que consienten, por lo demás, la inclusión de indicaciones geográficas como vías de asociar a los productos o servicios ofertados a la importante información transmitida por el signo, lo que, si bien constituye un instrumento concurrencial de interés, suele ser detonante sin embargo de interesantes conflictos.

\section{BILBIOGRAFÍA}

Abriani N., Cottino G., Ricolfi M., Diritto Industriale, Trattato di Diritto Comerciale, Vol. 2, Turín 2001.

Botana Agra M., Las denominaciones de origen, Madrid 2001.

Casado Cerviño A., La nueva Ley española de marcas, nombre comercial y rótulo de establecimiento. Razones para una reforma. Concepto de marca. Signos, "AC" 1990.

Fernández-Nóvoa C., Fundamentos de derecho de marcas, Madrid 1984.

Fernández-Nóvoa C., La adopción de un nombre geográfico extranjero como marca, "ADI" 1975, Vol. 2.

Fernández-Nóvoa C., La protección internacional de las denominaciones geográficas de los productos, Madrid 1970.

Fernández-Nóvoa C., Tratado de Derecho de Marcas, 2a edic., Madrid 2004.

Floridia G., Il riassetto della proprietà industriale, Milán 2006.

Ghidini G., Benedetti F. De, Codice della proprietà industriale. Commento alla normativa sui diritti derivanti da brevettazione e registrazione, $1^{\text {a }}$ edic., Milán 2006.

Gómez Lozano M.M., Denominaciones de origen y otras indicaciones geográficas, Cizur Menor 2004.

Gómez Segade J.A., La protección constitucional de la marca y de las denominaciones de origen, "ADI" 1981, Vol. 7.

Heaht C., Il caso Budweiser, "Riv. Dir. Ind.” 2004, Vol. 2-3.

Ivone G., Diritto alla denominazione e marchio geográfico, "Riv. Dir. Comm." 1993, Vol. 2.

Libertini M., Indicazioni geografiche e segni distintivi, "Riv. Dir. Comm." 1996, Vol. 1.

Magelli S., Marchio e nome geografico, [en:] Studi di Diritto Industriale in onore di Adriano Vanzetti. Proprietà intellettuale e concorrenza, Vol. 2, Milán 2004. 
Martínez Gutiérrez A., La conformación de la marca de empresa con denominaciones geográficas, "ADI" 1999, Vol. 20.

Massaguer Fuentes J., Marca (Derecho Mercantil), “EJB”, Vol. 3, Madrid 1995.

Mota Maia J., Marcas, indicaciones geográficas y denominaciones de origen en el derecho comunitario, [en:] A. Bercovitz Rodriguez-Cano, Marca y Diseño comunitarios, Cizur Menor 1996.

Nervi A., Le denominazioni di origine protette e marchi: spunti per una ricostruzione sistematica, "Riv. Dir. Comm." 1996, Vol. 1.

Plasseraud Y., Choisir, protéger et gerer vos marques, París 1977.

Ricolfi M., I fatti costitutivi del diritto al marchio. I soggetti, [en:] P. Auteri, G. Floridia, V. Mangini, G. Olivieri, M. Ricolfi, P. Spada, Diritto Industriale, Proprietà intellettuale e concorrenza, $2^{\mathrm{a}}$ edic., Turín 2005.

Sena G., Il diritto dei marchi. Marchio nazionale e marchio comunitario, $5^{\text {a }}$ edic., Milán 2007.

\section{STRESZCZENIE}

Chronione oznaczenia geograficzne stanowią dla podmiotów wprowadzających na rynek unijny produkty rolnicze formę prawną kompleksową i bezpieczną. Należy mieć jednak na uwadze, że ten sposób ochrony produktów rolno-żywnościowych z jednej strony oferuje wiele korzyści, w postaci np. możliwości reklamy swojego produktu na rynkach Unii Europejskiej, wyróżniając go spośród innych, z drugiej strony niekiedy komplikuje sytuację przedsiębiorcy. Celem niniejszego artykułu jest przedstawienie zalet i wad ochrony żywności za pomocą formy prawnej w postaci chronionych oznaczeń geograficznych.

Słowa kluczowe: oznaczenia geograficzne; reklama; ochrona 